\title{
Drug release from liposome coated hydrogels for soft contact lenses: the blinking and temperature effect
}

\author{
P. Paradiso ${ }^{1,2}$, R. Colaço ${ }^{1,2}$, J.L.G. Mata ${ }^{1}$, R. Krastev $^{3,4}$, B. Saramago ${ }^{1}$, A.P. Serro ${ }^{* 1,5}$
}

\footnotetext{
${ }^{1}$ Centro de Química Estrutural, Complexo I, Instituto Superior Técnico, University of Lisbon, Av. Rovisco Pais, 1049-001 Lisboa, Portugal

${ }^{2}$ Mechanical Engineering Department and IDMEC, Instituto Superior Técnico, University of Lisbon, Av. Rovisco Pais, 1049-001 Lisboa, Portugal

3 NMI - Natural and Medical Sciences Institute at the University of Tuebingen, Markwiesenstraße 55, 72770 Reutlingen, Germany

${ }^{4}$ Reutlingen University, D-72762, Reutlingen, Germany

${ }^{5}$ Centro de Investigação Interdisciplinar Egas Moniz, Instituto Superior de Ciências da Saúde Egas Moniz, Quinta da Granja, Monte de Caparica, 2829-511 Caparica, Portugal
}

*Correspondence to: A.P. Serro, Centro de Química Estrutural, Complexo I, Instituto Superior Técnico, University of Lisbon, Av. Rovisco Pais, 1049-001 Lisboa, Portugal. Email: anapaula.serro@tecnico.ulisboa.pt

Keywords: soft contact lenses; liposomes; drug release; eyelid; friction.

\begin{abstract}
In the present paper, liposome-based coatings aiming to control drug release from therapeutic soft contact lenses (SCLs) materials are analyzed. A PHEMA based hydrogel material loaded with levofloxacin (LVF) is used as model system for this research. The coatings are formed by polyelectrolyte layers containing liposomes of 1,2-dimyristoyl-snglycero-3-phosphocholine (DMPC) and DMPC+cholesterol (DMPC+CHOL). The effect of friction and temperature on the drug release is investigated. The aim of the friction tests is to simulate the blinking of the eyelid in order to verify if the SCLs materials coated with liposomes are able to keep their properties, in particular the drug release ability. It was observed that under the study conditions, friction did not affect significantly the drug release from the liposome coated PHEMA material. In contrast, increasing the temperature of release leads to an increase of the drug diffusion rate
\end{abstract}


through the hydrogel. This phenomenon is recorded both in the control and in the coated samples.

\section{Introduction}

Nowadays eye diseases are treated, in $90 \%$ of the cases, through the use of topical eye drops in form of solution or suspensions ${ }^{1}$. Eye drops instillations are well accepted by patients due to the cost-effectiveness and to the easy application; however, these conventional ocular formulations show low bioavailability due to physiological and anatomical constraints of the eye. Only a small fraction of the administered dose is absorbed $(<5 \%)^{2}$ due to: the continuous tear dilution ${ }^{3,4}$, dispersion and drainage during blinking and tearing reflex ${ }^{1,3}$, and due to the non-specific absorption ${ }^{3,5}$. Given the limited tear volume capacity of the eye, $\approx 7 \mu \mathrm{L}^{6}$, a large part of the drug dose $(25 \mu \mathrm{L}$ per drop) is squeezed out immediately after the eye drop is instilled. Additionally, after instillation, the drainage of the tear increases ${ }^{2}$, taking 5-10 minutes, to reset the volume to the normal physiological conditions ${ }^{7,8}$. As a result of the tear drainage, part of the drug is absorbed into the systemic circulation directly in the local blood capillaries of the conjunctival sac or in the nasal cavity ${ }^{9}$, reaching all major organs, with eventual side effects ${ }^{10}$. Furthermore, another fraction of the drug instilled is absorbed by the conjunctiva which has a larger area and higher permeability than the cornea ${ }^{11,12}$. High drug loss and poor cornea's absorption result in multiple dosing and extended eye drops therapies. The high instillation frequency reduces the patient compliance and, furthermore, may lead to drug overdosing ${ }^{13}$.

For these reasons, new ophthalmic drug delivery vehicles have been investigated, aiming at the design of a product which is, at the same time, capable to perform drug controlled release, without affecting vision and eye functions (blinking and tearing), and whose production must be cost effective and approved by regulatory terms. Within this context, soft contact lenses (SCLs) have emerged as a valid option for drug delivery to the anterior chamber of the eye, due to their high degree of comfort, biocompatibility and prolonged contact with the eye. Drug-loaded SCLs, may have a continuous therapeutic effect if providing a sustained drug release. When a drug loaded contact lens 
is placed on the eye, the drug diffuses through the lens matrix, and enters the PoLTF (post lens tear film), where drug molecules, thanks to the presence of the lens, will have a longer residence time compared to the case of topical application as drops ${ }^{5,14,15}$.

SCLs materials can be loaded with drugs by soaking. Although this is a simple and inexpensive method, which leads to a larger drug uptake by the ocular tissues than topical application of drops, it still may be ineffective, since drug is quickly released, generally with an initial burst. To enhance the drug loading capability and achieve a controlled drug release, several strategies may be attempted. The present work focuses on the study of the effect of the liposome coating on the drug release behavior.

Liposomes are spherical self-closed structures, composed of curved phospholipids bilayers. Thanks to the amphiphilic character of the phospholipids, liposomes are characterized by their biocompatibility and by their capacity to incorporate hydrophilic and hydrophobic drugs 16,17. For these reasons, these arrangements have received widespread attention as carrier system for therapeutically active compounds ${ }^{18}$. In recent years researchers presented studies in which the presence of liposomes, dispersed in a hydrogel matrix ${ }^{19}$, or immobilized on the contact lens surface ${ }^{20,21}$, enabled for a prolonged ophthalmic drug release from SCLs.

According to Skirtach et al. ${ }^{22}$, polyelectrolyte films showed the capacity of incorporation of nano and micro carriers, such as liposomes or polymeric capsules. Volodkin et al. proposed biocoatings for implant materials consisting in liposomes incorporated in polyelectrolyte coatings ${ }^{23,24}$ assembled using the layer-by-layer (LbL) technique, first proposed by Decher in $1991^{25}$.

As far as the authors know, all the studies presented in the literature which investigate coatings with liposomes to control the drug release from SCLs materials, use drug loaded liposomes. None of them focus on the use of bare liposomes immobilized on the surface of drug loaded SCL hydrogels to act as barriers to the drug release. Furthermore, the biotribological effect of the eyelid blinking on the stability of the liposome coatings and, consequently on their ability to control drug release also was never investigated.

In this work a coating formed by polyelectrolyte layers with additional adsorbed layer of liposomes was used as a tool to control the drug release from a HEMA/PVP material previously loaded with a model drug. Two lipid compositions were used for the 
liposomes preparation: 1,2-dimyristoyl-sn-glycero-3-phosphocholine (DMPC) and DMPC+cholesterol (DMPC+CHOL). The presence of cholesterol in the liposome formulation is known to improve the viscoelastic properties of the liposomes, as described in Serro et al. ${ }^{26}$. Through this protocol, the long immobilization process proposed by Danion et al. ${ }^{21}$ was avoided. Levofloxacin (LVF), a broad-spectrum antibiotic of the fluoroquinolone drug class, was used as model drug for this study.

The morphology of the polyelectrolyte+liposome coated surfaces were analyzed by atomic force microscopy (AFM). The polyelectrolyte bilayer and the lipid barrier formation were confirmed with the Quartz Crystal Microbalance with dissipation (QCM-D). Confocal fluorescence microscopy was used to study the lipid structure in the coating and their integrity upon LbL immobilization.

Especially designed and conceived apparatus to simulate the blinking movement of the eyelids was used to study the influence of repetitive load and friction cycles associated with blinking on the drug release profiles of PHEMA coated with liposomes.

In order to study the influence of the transition temperature of the lipids $\left(T_{t}\right)$ on the eventual coating barrier effect, the LVF release profiles from the PHEMA based hydrogel were obtained in static sink conditions at different temperatures (4, 20 and $\left.35^{\circ} \mathrm{C}\right)$. The effect of the lipid barrier on the drug release was studied through the comparison of the LVF release profiles from the coated and non-coated samples of PHEMA based hydrogel.

\section{Experimental Methods}

\subsection{Materials}

2-Hydroxyethyl methacrylate (HEMA), ethylene glycol dimethacrylate (EGDMA), 2,2'azobis(2-methylpropionitrile) (AIBN), levofloxacin (LVF), phosphoric acid, triethylamine, the buffer $\mathrm{N}$-(2-hydroxyethyl) piperazine- $\mathrm{N}^{\prime}$-(2-ethanesulfonic acid) (HEPES), chloroform, dichloromethane, and the polyelectrolytes: polyethylenimine (PEI) with mean molecular weight of $750 \mathrm{KDa}$, poly(sodium 4-styrene-sulfonate) (PSS) 
with mean molecular weight of $70 \mathrm{KDa}$, poly(allylamine hydrochloride) (PAH) with mean molecular weight of $70 \mathrm{KDa}, 5,6$-carboxyfluorescein $(\mathrm{CF})$ were all purchased from Sigma-Aldrich. Poly(vinylpyrrolidone) (PVP K30, Kollidon® 30) was kindly provided by BASF. Octadecyl Rhodamine B Chloride (R18) was from Invitrogen (Carlsbad, CA, USA). The lipids 1,2-Dimyristoyl-sn-glycero-3-phosphocholine (DMPC), and cholesterol (CHOL) were obtained from Avanti Polar Lipids (Alabaster, AL, USA). Sodium chloride was obtained from Merck, carbon tetrachloride from Riedel-de Haën, acetonitrile from Fisher Scientific, and dimethyldichlorosilane from Fluka. Solutions of Hellmanex II 2\% (Hellma $\mathrm{GmbH}$ ) and sodium dodecyl sulfate (SDS, Sigma-Aldrich) 3\% were used to clean the QCM-D parts and the quartz crystals, respectively. The AT-cut $5 \mathrm{MHz}$ gold piezoelectric quartz crystals (14 $\mathrm{mm}$ in diameter) (Q-Sense, Gothenburg, Sweden) were used in the QCM experiments. DD water was used in all experiments.

\subsection{Hydrogel preparation}

Conventional HEMA based hydrogel, HEMA/PVP (98/2, w/w) was prepared as follows. An appropriate amount of the crosslinker EGDMA was dissolved in HEMA (hydrophilic monomer) to obtain a concentration of $80 \mathrm{mM}$. Then, the mixture was degassed by ultra-sounds ( $5 \mathrm{~min}$ ) and bubbled with a gentle stream of nitrogen (15 $\mathrm{min}$ ) before the addition of AIBN (initiator), final concentration $10 \mathrm{mM}$, and PVP (hydrophilic additive), final concentration of $0.02 \mathrm{~g} / \mathrm{mL}$. The solution was magnetically stirred for about two hours to obtain complete dissolution of PVP. The mixture was then degassed by ultra-sounds ( $5 \mathrm{~min}$ ) and bubbled with a gentle stream of nitrogen (15 $\mathrm{min})$ before the final addition of AIBN to obtain a concentration of $15 \mathrm{mM}$. The final mixture was magnetically stirred for about $10 \mathrm{~min}$, to obtain a homogeneous solution. The mixture was injected into a mould consisting of two silanized glass plates separated by a spacer of polyurethane or teflon. The glass plates were silanized according to the procedure described by Vasquez et al $^{27}$, in order to facilitate the hydrogel removal from the mould. Briefly, the glasses were incubated in a $2 \%$ solution of dimethyldichlorosilane in carbon tetrachloride for one hour and then rinsed with dichloromethane and dried with nitrogen. The polymerization reaction was performed at $50{ }^{\circ} \mathrm{C}$ for 14 hours, followed by 24 hours at $70^{\circ}$. The obtained hydrogel sheets were 
washed over 5 days, with DD water renewed 3 times a day, to remove unreacted monomers and to facilitate the cutting of the samples used in the study. The hydrated HEMA/PVP hydrogel samples with a thickness of $0.30-0.35 \mathrm{~mm}$ were cut in pieces of $50 \times 10 \mathrm{~mm}^{2}$, and dried inside an oven at $40{ }^{\circ} \mathrm{C}$ overnight.

The main properties of HEMA/PVP hydrogel were fully characterized and reported in a previous published work ${ }^{28}$. The hydrogel demonstrated to present adequate characteristics to be used as ophthalmic material.

\subsection{Drug loading}

The dry HEMA/PVP hydrogel samples were loaded with LVF by soaking in the drug solution $\left(2.6 \mathrm{~mL} / \mathrm{cm}^{2}\right.$ of surface area), with a concentration of $5 \mathrm{mg} / \mathrm{mL}$ for 14 hours at $4{ }^{\circ} \mathrm{C}$. Levofloxacin was dissolved in saline solution $(130 \mathrm{mM} \mathrm{NaCl})$. The soaking process was performed protecting the loading solution from light. The loaded samples were rinsed with DD water and blotted with absorbent paper.

\subsection{Liposomes production}

Two types of liposomes were prepared, namely DMPC and DMPC/CHOL liposomes. Appropriate amounts of DMPC and DMPC + CHOL (70:30 mol\%) were dissolved in chloroform, and then dried under a nitrogen stream. The resulting film was kept under vacuum for at least 3 hours in order to remove all traces of organic solvent. After drying, the film was hydrated with HEPES (final concentration $5 \mathrm{mg} / \mathrm{mL}$ ) inside a thermostated water bath at $\approx 10-15{ }^{\circ} \mathrm{C}$ above the temperature of the gel-to-liquid crystalline phase transition. Heating was alternated with vortex agitation for 1 hour. The obtained multilamellar vesicles (MLVs) were submitted to 5 freezing-thawing cycles, respectively, in liquid nitrogen and in a water bath at the temperature referred above. Large unilamellar vesicles were obtained from the MLVs by extrusion in a stainless steel homemade extruder, thermostated at the same temperature. The samples were passed several times through polycarbonate filters (Nucleopore, Whatman) of decreasing pore size (600 nm, 5 times; $200 \mathrm{~nm}, 5$ times; and $100 \mathrm{~nm}, 10$ times), under 
inert nitrogen atmosphere, giving rise to small unilamellar vesicles. The liposome dispersions were stored at $4{ }^{\circ} \mathrm{C}$ and were used within 2 weeks from preparation. Liposome suspension of $0.7 \mathrm{mg} / \mathrm{mL}$ was prepared for the coating on HEMA/PVP hydrogel and for the QCM-D experiments. The choice of this concentration was based on a previous observations that this concentration leads to a monolayer of adsorbed liposomes on the quartz crystals ${ }^{26}$. Size distribution of extruded vesicles was determined at $25^{\circ} \mathrm{C}$ by dynamic light scattering (DLS) using a Spectra Physics model $127 \mathrm{He}-\mathrm{Ne}$ laser $(632.8 \mathrm{~nm}, 35 \mathrm{~mW})$ and a Brookhaven instrument with a BI-200SM goniometer, a BI-2030AT autocorrelator and a APD detector. For the confocal microscopy experiments, liposomes were prepared with the hydrophobic R18-labeled lipids and the encapsulated hydrophilic CF following a previously established protocol

29 . Lipids were labelled with R18, by adding an appropriate amount of dye to the mixture of lipids in chloroform, to reach a final concentration on $294 \mu \mathrm{M}$ in the final liposome suspension. To encapsulate CF inside the liposomes, the lipid film was hydrated in a solution of HEPES containing $50 \mu \mathrm{M}$ of the dye.

\subsection{Coatings assembly}

The polyelectrolyte multilayer coating on drug loaded HEMA/PVP hydrogel samples was prepared using the LbL technique of electrostatically driven sequential adsorption of polyions from their solutions ${ }^{30}$. Adsorption of polyelectrolytes was performed by immersion of the samples in the respective solutions; every solution had a concentration of LVF of $5 \mathrm{mg} / \mathrm{mL}$, except the liposome suspension. PEI solution had a concentration of $10 \mathrm{mg} / \mathrm{mL}$ and $\mathrm{pH}$ adjusted to 7 with hydrochloric acid. Both PSS and PAH solutions had a concentration of $2 \mathrm{mg} / \mathrm{mL}$ in $0.5 \mathrm{M} \mathrm{NaCl}$ solution. PEI deposition step lasted 15 minutes, PSS and PAH deposition steps lasted 6 minutes. Rinsing in between the steps was done for 5 minutes in water. The liposomes deposition step lasted 20 minutes. The sequence of the depositions is shown in Figure 1.

Control samples were prepared without coating but undergoing immersion in drug solution for the same time as the coated samples during the coating assembly. 


\subsection{Characterization Techniques}

\subsubsection{QCM-D measurements}

QCM-D measurements were performed using the quartz crystal microbalance with dissipation (QCM-D, Q-Sense model E4) as described by Rodahl et al. ${ }^{31}$, using gold crystals. The piezoelectric crystals were excited at their fundamental resonance frequency and the third, fifth, seventh and ninth overtones have been observed. The decrease in the resonance frequency, $\Delta f / n$, describes an increase of the mass adsorbed to the crystal, while the change in dissipation, $\Delta D$, gives information on the viscoelastic properties, namely the dissipation factor, $D$, is related to the acoustic energy loss by the adsorbed film. In this work the reported changes in dissipation and in the normalized frequency refer to the third overtone of the crystal fundamental resonance frequency. From the timedependence of the frequency shift and of the dissipation change, the kinetics of the coating adsorption and the interaction between layers were investigated. The same polyion solutions and liposome suspension, used for the coating formation on hydrogels, were used for the QCM-D experiments. DD water (baseline), PEI solution, water (rinsing), PSS solution, water (rinsing), liposome suspension, water (rinsing), PSS solution and water (rinsing) were sequentially injected into the QCM-D cells. All injections lasted approximately 10 minutes under a flow rate of $0.1 \mathrm{~mL} / \mathrm{min}$, followed by a pause in order to allow for the stabilization of the frequency and dissipation signals. The experiments were performed at $35{ }^{\circ} \mathrm{C}$ and the results are averages of, at least, four independent measurements. Prior to each experiment, all the QCM-D balance parts were washed with Hellmanex solution, abundantly rinsed with DD water and finally dried through the blow of nitrogen. The gold crystals were sonicated 5 minutes in SDS solution, abundantly rinsed with DD water and sonicated twice for 5 minutes in DD water, and finally blown dried with nitrogen. Immediately before the QCM-D experiments, the crystals were subjected twice to UV/ozone treatments (10 minutes), rinsing with DD water and drying with nitrogen flow.

\subsubsection{Surface topography}

The topography of the coatings on HEMA/PVP hydrogels was examined by AFM. Different samples were prepared for each step of the coating, namely: after the first 
polyanion bilayer (PEI/PSS), after the liposomes layer ((PEI/PSS/ DMPC or DMPC /CHOL liposomes) and finally, after the second bilayer (PEI/PSS/liposomes/PSS/PAH). The samples were maintained hydrated and a Nanosurf AFM was used in contact mode in liquid $(130 \mathrm{mM} \mathrm{NaCl})$, at $25^{\circ} \mathrm{C}$, with a gold coated PPP-CONTSCAuD Nanosensor cantilever (force constant $0.06 \mathrm{~N} / \mathrm{m}$ ). All observations were conducted at $25{ }^{\circ} \mathrm{C}$. The average roughness was determined from at least five regions in the AFM images.

\subsubsection{Confocal fluorescence microscopy}

Fluorescence images of the liposome coatings before and after the polyelectrolyte bilayer addition, were collected using a confocal fluorescence Zeiss Axiovert 200M using a 63x water immersion objective (high magnification). The images were analysed with QWin software (Leica Microsystems, Heidelberg, Germany). The fluorescence imaging of the coatings permitted to better understand the liposomes coating adherence and interaction with the polyelectrolyte layers. Hydrogels could not be used as substrates because CF was absorbed by the matrix, overlapping the fluorescence signal of the liposomes. Silicon oxide samples served as substrate for the coatings which were prepared following the same procedure as described in section 5.2.4. Liposomes with both compositions (DMPC and DMPC/CHOL) were tested. The coatings were imaged, before and after the deposition of the second bilayer: PEI/PSS/liposomes and PEI/PSS/liposomes/PSS/PAH. After every step of the coating assembly the silicon surface was extensively rinsed with DD water.

\subsubsection{Transmittance}

Transparency studies were carried out measuring the percent transmittance of visible light (wavelength range from 400 to $700 \mathrm{~nm}$ ) through hydrated contact lenses previously coated with both liposomes composition (DMPC and DMPC+CHOL). The samples were directly mounted on the outer surface of a quartz cuvette, which was then placed in a spectrophotometer (UV-vis Beckmam DU-70). The tests were done in triplicate. Transmittance of hydrogel samples without coating was measured as control.

\subsection{Drug release}




\subsubsection{Experiments under simulated eyelid movement}

The effect of the eyelid movement on the drug release performance of uncoated PHEMA based hydrogels, was studied in a previous work by Serro et al. ${ }^{32}$, using the Simublink apparatus (see Figure 2). The working principle of Simublink resides on the conversion of the rotation motion of a stepping motor into alternate linear motion. An Arduino interface (Uno+EasyDriver) controls the motor pace. The contact pressure created by the eyelid during blinking was estimated to be in the range 3.5-4.0 $\mathrm{kPa}$ with a blinking speed average around $12 \mathrm{~cm} / \mathrm{s}^{33}$. Due to design limitations, a pressure of $16 \mathrm{kPa}$ was applied on the hydrogel using a cylinder shaped counter body of PMMA with a weight of $3.5 \mathrm{~g}$. The final pressure was calculated through the following formula ${ }^{34}$ :

$P_{\text {max }}=\left(\frac{E^{*}}{2 \pi R} * \frac{F_{N}}{L}\right)$

where $\mathrm{E}^{*}$ is the elastic modulus depending on the Young modulus and Poisson coefficient of both materials, $\mathrm{R}$ is the radius of the cylinder, $\mathrm{F}_{\mathrm{N}}$ the normal force and $\mathrm{L}$ the length of the contact area between the cylinder and the plane hydrogel.

A sliding velocity of $14 \mathrm{~cm} / \mathrm{s}$, making a 2 seconds pause between "blinkings" to mimic the eye conditions ${ }^{33}$.

Both lipid composition coatings, PEI/PSS/liposomes/PSS/PAH, with DMCP and DMPC + CHOL liposomes, were submitted to the friction experiments simulating the eyelid action. The experiments were performed not at the physiological ocular temperature of $35^{\circ} \mathrm{C}^{35}$, but at $20^{\circ} \mathrm{C}$ due to experimental limitations.

The experiments were done using strips of the hydrogels of $5 \times 1 \mathrm{~cm}^{2}$ and keeping the ratio for the supernatant volume $\left(2.6 \mathrm{~mL}\right.$ of $\mathrm{NaCl}$ solution $/ \mathrm{cm}^{2}$ of surface area). At predetermined time intervals, aliquots ( $~ 8 \%$ of total volume) of the supernatant were collected and replaced by the same volume of fresh $\mathrm{NaCl}$ solution. The concentration of LVF in the supernatant was determined using a high performance liquid chromatograph (HPLC), at the wavelength of $290 \mathrm{~nm}$, with a Jasco UV-VIS detector and a C-18 column Nova-Pak Watters. The mobile phase, which consisted of DD water, acetonitrile, phosphoric acid and triethylamine (86/14/0.6/0.3 in volume), was introduced into the column at a flow rate of $1 \mathrm{~mL} / \mathrm{min}$ and a pressure of $14 \mathrm{MPa}$. 
The experiments were performed in triplicate.

\subsubsection{Effect of temperature at static sink conditions}

Drug release experiments in static conditions were performed on coated and non-coated samples. The previously loaded and coated samples were immersed in the saline solution $\left(2.6 \mathrm{~mL} / \mathrm{cm}^{2}\right.$ of surface area), in closed vessels, under stirring (150 rpm). At pre-determined time intervals, aliquots ( $\sim 8 \%$ of total volume) of the supernatant were collected and replaced by the same volume of fresh $\mathrm{NaCl}$ solution. The concentration of LVF in the supernatant was determined as described before. In the case of DMPC liposomes, the effect of temperature of release on the behavior of the lipid coating was investigated. As the gel-liquid crystalline phase transition temperature, $\mathrm{T}_{\mathrm{t}}$, of DMPC is $23.7{ }^{\circ} \mathrm{C}{ }^{36}$, three different temperatures were chosen: two temperatures below $\mathrm{T}_{\mathrm{t},} 4{ }^{\circ} \mathrm{C}$ and $20^{\circ} \mathrm{C}$, and one above, $35^{\circ} \mathrm{C}$.

The experiments were performed in triplicate.

\section{Results and discussion}

\subsection{Coating characterization}

The formation of the liposome-based coating on gold coated quartz crystals was followed using QCM-D. The average size of the liposomes was determined to be $103 \pm 8 \mathrm{~nm}$. The formation of the precursor PEI layer on the HEMA/PVP hydrogels was confirmed by fluorescence tests not shown in this paper.

The frequency and dissipation shifts for the third overtone, obtained during deposition, are shown in Figure 3. The polyelectrolytes deposition and the liposomes adsorption are accompanied by a decrease in the frequency signal which related to an increase in mass of the coating. The injection of the liposome suspension causes a large shift in the resonant frequency, $\Delta \mathrm{f} / \mathrm{n} \sim 350 \mathrm{~Hz}$, in both liposome compositions (Figure $3 \mathrm{~A}$ and $\mathrm{B}$ ), and a relative large change in dissipation, which confirms the adsorption of intact liposomes 37-39. The frequency and dissipation shifts for the other overtones (not shown) are not overlapped confirming the viscoelastic nature of the adsorbed layer. The frequency shift 
(third overtone) corresponding to the adsorption of both DMPC and DMPC+CHOL liposomes is higher than the one described in literature on clean gold ${ }^{37-39}$, which may be due to the presence of the polyelectrolytes underlayer. During rinsing with DD water, which follows each adsorption step (PEI, PSS and of the liposomes), there is an increase in frequency and a decrease in dissipation, which represents a compaction of the film and removal of weakly adsorbed species. After adsorption of the liposomes, during the injection of the PSS solution, the frequency strongly increases in the case of both lipids $(\Delta \mathrm{f} / \mathrm{n} \sim 150 \mathrm{~Hz})$, while the dissipation increases in the case of DMPC liposomes $(\Delta \mathrm{D} \sim 53$ $\times 10^{-6}$ ) (Figure $3 \mathrm{~A}$ ), and slightly decreases in the case of the DMPC+CHOL liposomes $\left(\Delta \mathrm{D} 30 \times 10^{-6}\right)$ (Figure 3B). These values are too high to hypothesize the total rupture of the liposomes and formation of a single bilayer, which, from literature, would correspond to $\Delta \mathrm{f} / \mathrm{n} \sim 25 \mathrm{~Hz}$ and $\Delta \mathrm{D} \sim 1 \times 10^{-6} 40,41$. Thus, it is plausible to assume partial rupture of the liposomes creating a heterogeneous layer where fragments of liposomes shall coexist with bilayer regions and eventually some intact liposomes. The increase in dissipation observed for DMPC seems to indicate that, in this case, the layer became more fluid. During the following rinsing step, the frequency is not affected, while the dissipation decreases in both cases, in a stronger way in the case of the DMPC lipids. After PAH adsorption, during the DD water rinse, dissipation values do not change, but the frequency increases. This behaviour may be justified by the detachment of weakly bounded molecules, without any change in the viscoelastic properties.

Contact mode AFM images of coated HEMA/PVP samples are presented in Figure 4. All observations were done in an aqueous environment with swollen samples. The AFM images shown are representative of the samples. The adsorption of the polyelectrolyte forms heterogeneities (spots with aggregates) on the surface. The average roughness of the samples is reported in the figures. The roughness of the surface increases with the adsorption of the liposomes and decreases with the subsequent adsorption of the polyelectrolytes bilayer.

Confocal fluorescence microscopy investigation was used to further investigate the stability and the structure of the DMPC and DMCP+CHOL liposomes. As described in the experimental part, liposomes were labelled with fluorescent dyes, namely the hydrophilic CF encapsulated inside the liposomes and R18 labelling the lipid layers. Two types of samples were prepared for these measurements, representing two steps of the 
coating, namely, the liposomes layer before and after the PSS/PAH polyelectrolytes bilayer adsorption.

Figure 5 shows the fluorescent emission of CF (obtained at 530-550 nm) and of R18 (obtained at 580-680nm) on the coated silicon samples. Figure 5A presents the fluorescent emissions of the adsorbed liposomes. Both fluorescent emissions of $\mathrm{CF}$ and $\mathrm{R} 18$ are visible in the figures in both DMPC and DMPC+CHOL coatings, evidencing the presence of intact liposomes. However, the signal is not homogeneous, indicating a discontinuous coating of the surface, which is in agreement with AFM observations. After the PSS/PAH polyelectrolytes bilayer adsorption (Figure 5B), in the case of DMPC, the fluorescent signal of the encapsulated CF is not recorded, being a sign of liposomes rupture. This outcome is in contradiction with the previously described QCM results, and it is probably consequence of resolution limitations of the fluorescence microscopy. In the case of $\mathrm{DMCP}+\mathrm{CHOL}$, the sample presents areas where the $\mathrm{CF}$ signal is still emitted and other areas without signal. These observations are in agreement with the QCM-D results: the higher stiffness and viscosity of the DMPC+CHOL liposomes suggests that they are more resistant to rupture because the lipids are more densely packed, as described in Serro et al. ${ }^{26}$.

The transmittance of the coated hydrogel with both types of liposome (DMPC and $\mathrm{DMPC}+\mathrm{CHOL}$ ) was not significantly affected relatively to the control sample; all the measured samples have over $90 \%$ transparency, matching the minimum transmittance value required for soft contact lenses ${ }^{42}$. In detail, the DMPC coating decreases the hydrogel transmittance from $99.0 \pm 0.2 \%$ to $98.7 \pm 0.3 \%$ while the DMPC+CHOL coating reduces the transmittance to $97.6 \pm 0.4 \%$.

\subsection{Drug Release}

Figure 6 compares the cumulative release curves of LVF obtained in static sink conditions with those obtained under friction conditions which attempt to simulate the eye blinking effect. The experiments were carried out at $20^{\circ} \mathrm{C}$. At this temperature, both lipids, DMPC and DMPC + CHOL, are in the gel phase, since the $\mathrm{T}_{\mathrm{t}}$ of DMPC is $23{ }^{\circ} \mathrm{C}$ and of DMPC $+\mathrm{CHOL}$ is $30^{\circ} \mathrm{C}^{43}$. 
In a previous work performed by Serro et al. ${ }^{32}$, the authors concluded that friction does not influence significantly the LVF release of thermal polymerized HEMA/PVP hydrogels, which is confirmed by the data presented here.

Concerning the coated samples, it can be seen that both coatings play a barrier effect, reducing the amount of drug released, but do not affect significantly the release kinetics. No noticeable difference between the curves obtained with and without friction could be observed, indicating that both coatings maintain their protective effect.

The average coating thickness is expected to be within the order of $100 \mathrm{~nm}$. In fact, although the diameter of the vesicles in suspension is about $100 \mathrm{~nm}$, they suffer some flattening upon adsorption ${ }^{44}$. Furthermore, as it was demonstrated by the QCM analysis, partial rupture of the liposomes occurs. Finally, the thickness of the polyelectrolyte layers shall not be higher than $20 \mathrm{~nm}$ (10nm/bilayer) ${ }^{45}$. Considering a total thickness of $200 \mathrm{~nm}$ (taking into account both sides of the hydrogel coating), it would only correspond to $\sim 0.07 \%$ of the total thickness of the hydrogel (300 $\mu \mathrm{m}$ thick). The relative small thickness of the coating suggests that the control mechanism of drug transport through the liposome coating is not simply Fick's diffusion, but depends on the interactions between the amphiphilic drug and the lipid coating.

The effect of temperature of release on the cumulative release profiles of LVF obtained in static sink condition was also investigated. Although the release in vivo occurs around $35^{\circ} \mathrm{C}$, the investigation on the effect of the temperature has the objective of elucidating if the release is affect by the lipid phase. In other words, the drug release was assessed above and below the lipid transition temperature. The results obtained with and without the PEI/PSS/DMPC liposomes/PSS/PAH coating assembly are shown in Figure 7.

Increasing the temperature of release leads to an increase of the drug diffusion rate through the hydrogel. This phenomenon is recorded both in the control and in the coated samples.

The effect of the presence of the coating on the release of levofloxacin is null at $4^{\circ} \mathrm{C}$, but increases with temperature, probably due to the phase transition that occurs in the DMPC bilayer at $23^{\circ} \mathrm{C}^{36}$. The liquid disordered state of the bilayer at $35^{\circ} \mathrm{C}$ may be responsible for the higher capacity of the lipids to decrease the drug release. However, it is important 
to stress that the kinetics of drug release is not much affected, in contrast to the amount of drug released that is significantly reduced.

\section{Conclusions}

In the present chapter the potential influence of liposome-based coatings on the improvement of the drug release performances of a PHEMA based hydrogel was studied.

The PHEMA based hydrogel was first loaded with levofloxacin (LVF) and subsequently coated with a polyelectrolyte coating containing liposomes. Two liposome compositions were tested, DMPC and DMPC+CHOL, which give rise to coatings with different viscoelasticity: DMPC+CHOL liposomes confer higher viscosity and stiffness to the coating. The coatings were characterized using different techniques: QCM-D, AFM and confocal fluorescence microscopy. It was demonstrated that after the intact liposome adsorption, there is partial rupture of the liposomes caused by the interaction with the anionic polyelectrolytes. From the drug release experiments performed in the presence of friction, which simulate the eye blinking on the SCLs during wear, it was concluded that no noticeable difference is introduced by the presence of friction, either for the control or the coated samples.

Both coatings lead to a decrease on the amount of drug released, but do not affect significantly the release rate. Different temperatures of release were tested for the samples coated with DMPC liposomes, namely 4, 20 and $35^{\circ} \mathrm{C}$ in order to study the influence of the $T_{t}$ of the lipid. The results obtained showed that the drug release rate increased for higher temperatures due to the higher disorder degree of the lipid and expected increase in drug diffusion coefficient. The presence of the coating seems to retain part of the loaded drug, being this effect more visible with the increase of the temperature of release.

In conclusion, although in this particular case, friction does not affect the drug release behavior of the SCLs materials; its effect must be evaluated in order to approach the eye conditions. Depending on the systems characteristics, it may be a relevant factor. 


\section{Acknowledgments}

To Fundação para a Ciência e a Tecnologia for Patrizia Paradiso PhD Grant (SFRH/BD/71990/2010), to the funding through the projects UID/QUI/00100/2013, MERA.NET/0005/2012 and the Luso-German Integrated Actions 2012 reference A-29/12. 


\section{Figure captions}

Figure 1. Schematic representation of the coating assembly steps

Figure 2. Simublink apparatus. 1) Experiment cell, 2) Power supply, 3) Arduino controller, 4) Step by step motor. In detail the top and side view (A) of the experimental cell.

Figure 3: Coating assembly monitored by QCM-D: A) PSS/DMPC liposomes/PSS/PAH;

B) PSS/DMPC/CHOL liposomes/PSS/PAH. The third overtone is shown.

Figure 4: Contact-mode AFM images of HEMA/PVP after deposition of PEI/PSS, PEI/PSS/liposomes and PEI/PSS/liposomes/PSS/PAH. The mean roughness values are reported.

Figure 5. Confocal fluorescent images of DMPC and DMPC/CHOL liposome coating before (A) and after (B) the polyelectrolyte bilayer adsorption. The fluorescent emission of CF (green) and of R18 (red) are reported. The images were obtained at x63 magnification.

Figure 6. Effect of the friction on the cumulative release profiles of levofloxacin from HEMA/PVP hydrogels controls and coated samples with A) PSS/DMPC liposomes/PSS/PAH layer and B) PSS/DMPC/CHOL liposomes/PSS/PAH.

Figure 7: Effect of the temperature of release on the cumulative release profiles of levofloxacin from HEMA/PVP hydrogels coated with a PSS/DMPC liposomes/PSS/PAH layer. 


\section{References}

1. Ghate D, Edelhauser HF. Ocular drug delivery. Expert Opin Drug Deliv 2006;3(2):275-87.

2. Gaudana R, Ananthula HK, Parenky A, Mitra AK. Ocular drug delivery. AAPS J 2010;12(3):348-60.

3. Chrai SS, Makoid MC, Eriksen SP, Robinson JR. Drop size and initial dosing frequency problems of topically applied ophthalmic drugs. J Pharm Sci 1974;63(3):333-8.

4. Friedlaender MH, Breshears D, Amoozgar B, Sheardown H, Senchyna M. The dilution of benzalkonium chloride (BAK) in the tear film. Adv Ther 2006;23(6):835-41.

5. Bourlais CL, Acar L, Zia H, Sado PA, Needham T, Leverge R. Ophthalmic drug delivery systems--recent advances. Prog Retin Eye Res 1998;17(1):33-58.

6. Larke J. Tears. In: Larke L, editor. The eye in contact lens wear. Boston: Butterworth Heinemann, Oxford; 1995. p 30.

7. Yokoi N, Komuro A. Non-invasive methods of assessing the tear film. Exp Eye Res 2004;78(3):399-407.

8. Zhu H, Chauhan A. A mathematical model for ocular tear and solute balance. Curr Eye Res 2005;30(10):841-54.

9. Urtti A. Challenges and obstacles of ocular pharmacokinetics and drug delivery. Adv Drug Deliv Rev 2006;58(11):1131-5.

10. Salminen L. Review: systemic absorption of topically applied ocular drugs in humans. J Ocul Pharmacol 1990;6(3):243-9.

11. Prausnitz MR, Noonan JS. Permeability of cornea, sclera, and conjunctiva: a literature analysis for drug delivery to the eye. J Pharm Sci 1998;87(12):1479-88.

12. Watsky MA, Jablonski MM, Edelhauser HF. Comparison of conjunctival and corneal surface areas in rabbit and human. Curr Eye Res 1988;7(5):483-6.

13. Trawick AB. Potential systemic and ocular side effects associated with topical administration of timolol maleate. J Am Optom Assoc 1985;56(2):108-12. 
14. McNamara NA, Polse KA, Brand RJ, Graham AD, Chan JS, McKenney CD. Tear mixing under a soft contact lens: effects of lens diameter. Am J Ophthalmol 1999;127(6):659-665.

15. Creech JL, Chauhan A, Radke CJ. Dispersive mixing in the posterior tear film under a soft contact lens. Ind Eng Chem Res 2001;40(14):3015-3026.

16. Schwendener RA, Schott H. Liposome formulations of hydrophobic drugs. Methods Mol Biol 2010;605:129-38.

17. Jaafar-Maalej C, Diab R, Andrieu V, Elaissari A, Fessi H. Ethanol injection method for hydrophilic and lipophilic drug-loaded liposome preparation. J Liposome Res 2010;20(3):228-243.

18. Mufamadi MS, Pillay V, Choonara YE, Modi G, Naidoo D, Ndesendo VMK. A Review on Composite Liposomal Technologies for Specialized Drug Delivery. J Drug Deliv 2011;2011:939851-70.

19. Gulsen D, Li CC, Chauhan A. Dispersion of DMPC liposomes in contact lenses for ophthalmic drug delivery. Current Eye Research 2005;30(12):1071-1080.

20. Danion A, Brochu H, Martin Y, Vermette P. Fabrication and characterization of contact lenses bearing surface-immobilized layers of intact liposomes. J. Biomed. Mater. Res. A. 2007;82(1):41-51.

21. Danion A, Arsenault I, Vermette P. Antibacterial activity of contact lenses bearing surface-immobilized layers of intact liposomes loaded with levofloxacin. J Pharm Sci 2007;96(9):2350-63.

22. Skirtach A, Volodkin D, Mohwald H. Remote and SelfInduced Release from Polyelectrolyte Multilayer Capsules. In: Decher G, Schlenoff J, editors. Multilayer Thin Films: Sequential Assembly of Nanocomposite Materials. Darmstadt, Germany: John Wiley \& Sons; 2012. p 838-940.

23. Volodkin D, Mohwald H, Voegel J-C, Ball V. Coating of negatively charged liposomes by polylysine: Drug release study. J Control Release 2007;117(1):111120.

24. Volodkin D, Arntz Y, Schaaf P, Möhwald H, Voegel JC. Composite multilayered biocompatible polyelectrolyte films with intact liposomes: stability and temperature triggered dye release. Soft Matter 2008;4(1):122-130.

25. Decher G, Hong JD. Buildup of Ultrathin Multilayer Films by a Self-Assembly Process: II. Consecutive Adsorption of Anionic and Cationic Bipolar Amphiphiles 
and Polyelectrolytes on Charged Surfaces. Berichte der Bunsengesellschaft für physikalische Chemie 1991;95(11):1430-1434.

26. Serro AP, Carapeto A, Paiva G, Farinha JPS, Colaço R, Saramago B. Formation of an intact liposome layer adsorbed on oxidized gold confirmed by three complementary techniques: QCM-D, AFM and confocal fluorescence microscopy. Surf and Interface Anal 2012;44(4):426-433.

27. Vazquez R, Nogueira R, Orfao M, Mata JL, Saramago B. Stability of triglyceride liquid films on hydrophilic and hydrophobic glasses. J Colloid Interface Sci 2006;299(1):274-82.

28. Paradiso P, Galante R, Santos L, Alves de Matos AP, Colaco R, Serro AP, Saramago B. Comparison of two hydrogel formulations for drug release in ophthalmic lenses. J Biomed Mater Res B Appl Biomater 2014.

29. Serro AP, Carapeto A, Paiva G, Farinha JPS, Colaço R, Saramago B. Formation of an intact liposome layer adsorbed on oxidized gold confirmed by three complementary techniques: QCM-D, AFM and confocal fluorescence microscopy. Surf Interface Anal 2012;44(4):426-433.

30. Multilayer Thin Films: Sequential Assembly of Nanocomposite Materials: WileyVCH; 2003.

31. Rodahl M, Höök F, Krozer A, Brzezinski P, Kasemo B. Quartz crystal microbalance setup for frequency and Q - factor measurements in gaseous and liquid environments. Review of Scientific Instruments 1995;66(7):3924-3930.

32. Galante RP, P, Moutinho M, Fernandes A, Mata J, Matos A, Colaço R, Saramago B, Serro A. About the effect of eye blinking on drug release from pHEMA-based hydrogels: an in vitro study. J Biomater Sci Polym 2015;26(4):235-51.

33. Rennie AC, Dickrell PL, Sawyer WG. Friction coefficient of soft contact lenses: measurements and modeling. Tribol Lett 2005;18(4):499-504.

34. Galante R. Influência da ação tribológica na libertação de levofloxacina a partir de materiais de lentes de contacto. Lisbon: Instituto Superior Tecnico; 2012.

35. Sniegowski M, Erlanger M, Velez-Montoya R, Olson JL. Difference in ocular surface temperature by infrared thermography in phakic and pseudophakic patients. Clinical Ophthalmology (Auckland, N.Z.) 2015;9:461-466.

36. Serro AP, Galante R, Kozica A, Paradiso P, da Silva AMPSG, Luzyanin KV, Fernandes AC, Saramago B. Effect of tetracaine on DMPC and 
DMPC+cholesterol biomembrane models: Liposomes and monolayers. Colloids Surf B 2014;116(0):63-71.

37. Stalgren JJ, Claesson PM, Warnheim T. Adsorption of liposomes and emulsions studied with a quartz crystal microbalance. Adv Colloid Interface Sci 2001;8990:383-94.

38. Seantier B, Breffa C, Felix O, Decher G. Dissipation-enhanced quartz crystal microbalance studies on the experimental parameters controlling the formation of supported lipid bilayers. J Phys Chem B 2005;109(46):21755-65.

39. Viitala T, Hautala JT, Vuorinen J, Wiedmer SK. Structure of anionic phospholipid coatings on silica by dissipative quartz crystal microbalance. Langmuir 2007;23(2):609-18.

40. Richter RP, Brisson AR. Following the formation of supported lipid bilayers on mica: a study combining AFM, QCM-D, and ellipsometry. Biophys J 2005;88(5):3422-33.

41. Keller CA, Glasmastar K, Zhdanov VP, Kasemo B. Formation of supported membranes from vesicles. Phys Rev Lett 2000;84(23):5443-6.

42. Efron N, Maldonado-Codina C. Development of Contact Lenses from a Biomaterial Point of View - Materials, Manufacture, and Clinical Application. In: Ducheyne P, editor. Comprehensive Biomaterials. Amsterdam: Elsevier Science; 2011. p 517-541.

43. Paiva JG, Paradiso P, Serro AP, Fernandes A, Saramago B. Interaction of local and general anaesthetics with liposomal membrane models: a QCM-D and DSC study. Colloids Surf B Biointerfaces 2012;95:65-74.

44. Jung H, Kim J, Park J, Lee S, Lee H, Kuboi R, Kawai T. Atomic force microscopy observation of highly arrayed phospholipid bilayer vesicle on a gold surface. J Biosci Bioeng 2006;102(1):28-33.

45. Feldoto Z, Varga I, Blomberg E. Influence of salt and rinsing protocol on the structure of PAH/PSS polyelectrolyte multilayers. Langmuir 2010;26(22):1704857. 